6 Lepage JY, Malinge $M$, Cozian A, Pinard M, Blanloeil $Y$, Sower $R$. Vecuronium and atracurium in patients with end stage renal failure. Br J Anaesth, 1987; 59: 1004-10.

\section{Epidural air-filled bubbles and unblocked segments}

To the Editor:

Dalens et al. ${ }^{1}$ pointed out that epidural air-filled bubbles can cause unblocked segments during epidural anaesthesia. We administered epidural anaesthesia to a 16-yearold primigravid patient for pain relief in labour, after which she experienced a persistent unblocked segment in the right groin. This was believed to be due to an air-filled bubble in the epidural space in the area of the nerve root supplying the right groin.

Loss of resistance to air was used to identify the epidural space after needle placement in the second lumbar interspace and a translucent multi-orificed catheter was inserted $4 \mathrm{~cm}$ into this space. Neither cerebrospinal fluid (CSF) nor blood could be aspirated from the catheter. A test dose of $2 \mathrm{ml}$ lidocaine two per cent with $1 / 200,000$ adrenaline was injected and was negative for catheter misplacement. Good obstetrical analgesia was achieved after $10 \mathrm{ml}$ of 0.25 per cent bupivacaine was injected. A segment in the right groin, however, remained unblocked. Top-ups were requested on two later occasions and at each biochemically proven CSF could be aspirated from the catheter. On each occasion $8 \mathrm{ml}$ bupivacaine 0.25 per cent fractionated in $2 \mathrm{ml}$ increments at five-minute intervals, was required to produce satisfactory obstetrical analgesia. The area in the right groin remained unblocked throughout. At no stage after the initial placement of the Tuohy needle was air injected into the epidural space.

Following the delivery and informed consent from the mother, $12 \mathrm{ml}$ of iopaminol ( $200 \mathrm{mg} \cdot \mathrm{ml}^{-1}$ ) was injected via the epidural catheter under fluoroscopic control. A radiograph of the spine (Figures $1 \mathrm{a}$ and $1 \mathrm{~b}$ ) revealed a normal peridurogram with a large air-filled bubble overlying the $T_{12}-L_{1}$ region on the right side. There was no evidence of subarachnoid spread of contrast medium.

We believe that the air-filled bubble in the $T_{12}-\mathrm{L}_{1}$ region was probably the cause of the unblocked segment in the corresponding dermatome in the groin. It can further be postulated that the normal peridurogram as well as the unexpected behaviour of the block following CSF aspiration, was due to the distal orifice of the multiorificed catheter migrating into the subarachnoid space

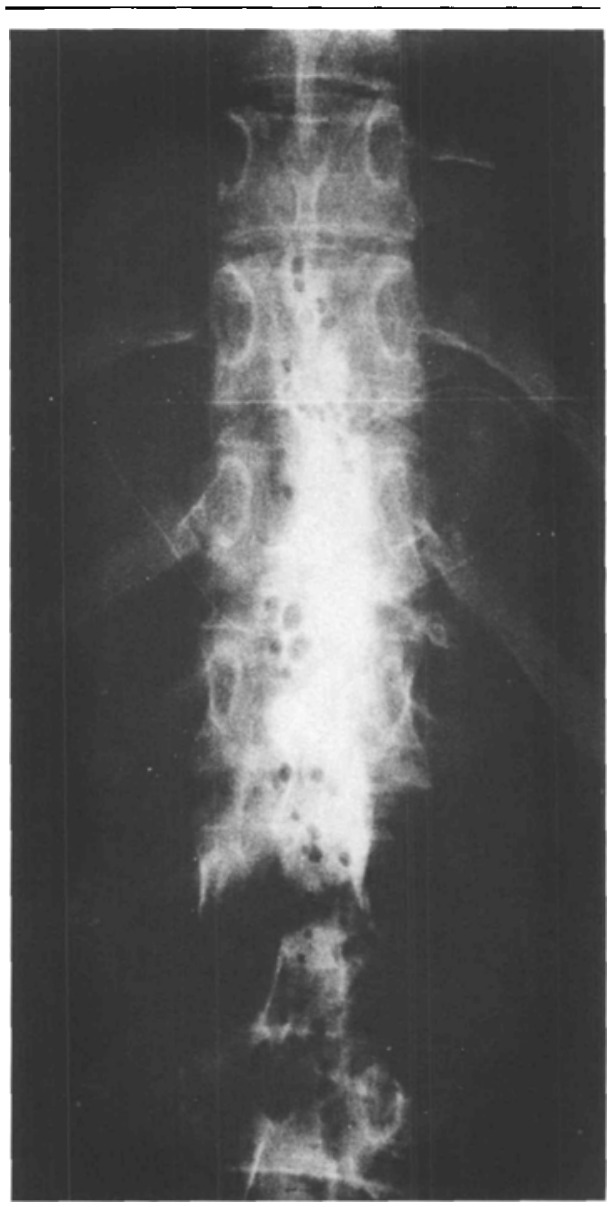

FIGURE la

while the proximal two orifices remained extradurally (Figure 2).

\section{Andre P. Boezaart}

MaChB MPrax Med, DA(SA) FFA(SA) MMed(Anaesth)

Bruce J. Levendig MBBCh DA(SA)

Department of Anaesthesia

University of the Witwatersrand

Johannesburg

Republic of South Africa 

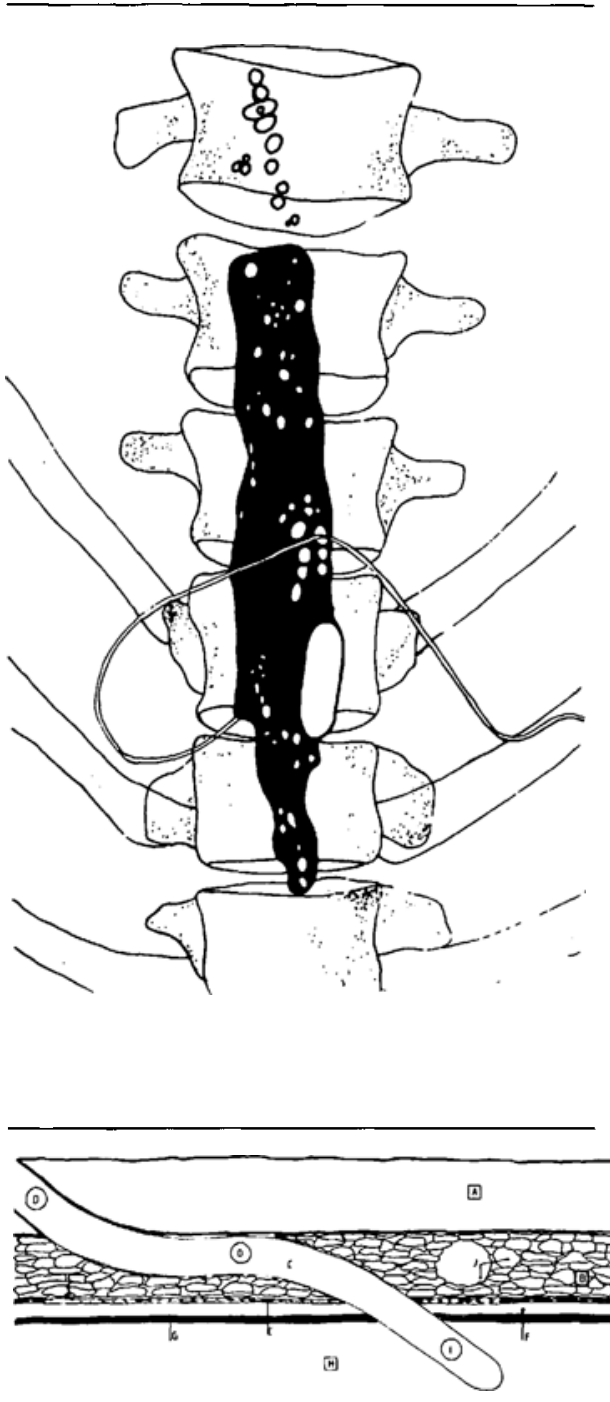

FIGURE 2 Schematic representation of epidural, subdural and subarachnoid space with catheter in situ. A - ligamentum flavum, B - epidural space, C - epidural catheter, D - proximal orifice in epidural space, E - dura mater, F - subdural space, G - arachnoid mater, $\mathrm{H}$ - subarachnoid space, $\mathbf{1}$ - distal orifice in subarachnoid space.

\section{REFERENCE}

1 Dalens B, Bazin J, Haberer J. Epidural bubbles as a cause of incomplete analgesia during epidural anesthesia. Anesth Analg 1987; 66: 679-83.

\section{Autonomic hyperreflexia dur- ing extracorporeal shock- wave lithotripsy (ESWL) in quadriplegic patients}

To the Editor:

Extracorporeal shock-wave lithotripsy (ESWL) is a widely used non-invasive method for treating patients with renal and ureteral calculi. ' Both general and regional anaesthesia have been used. When the procedure must be done in patients with spinal cord injury, it presents a special challenge to anaesthesists. ${ }^{2}$ We describe our experience with nine such patients managed with intravenous sedation and antihypertensive agents prn.

After approval from our institution's Human Studies Committee, a retrospective chart review was conducted for patients undergoing ESWL (Dornier System Company GmbH, Type HM3, 1983) between May 1985 and May 1986. Epidural anaesthesia was administered in 912/1225 cases ( 74.4 per cent) versus general anaesthesia in 230/1225 (18.8 per cent), and spinal anaesthesia in $73 / 1225$ (5.9 per cent). Nine quadriplegic patients were identified. Seven patients received one ESWL treatment apiece, whereas two patients received two treatments (Table). In ten of the 11 treatments, the patients had an increase in BP to over 140/90. Although five episodes were treated with vasodilating drugs, only in two episodes were the patients symptomatic with one patient complaining of headache and one patient complaining of anxiety. Anaesthesia for eight of 11 treatments was with IV sedation; two received no drugs; and one spinal anaesthetic was performed. Vital signs were stable in all patients when discharged from the recovery room back to their hospital room.

For the quadriplegic patient, ESWL is like any other surgical procedure in that blood pressure monitoring and physiological evaluation and support are extremely important. In our nine quadriplegic patients, we found that anaesthesia, monitoring and sedation (AMS) with treatment of hypertensive episodes to be adequate and safe for ESWL. The high recovery room blood pressures suggested that postoperative care is also important and 